\title{
Methods of Fault Diagnosis in Fiber Optic Current Transducer Based on Allan Variance
}

\author{
Lihui Wang, ${ }^{1,2}$ Gang Chen, ${ }^{3}$ Jianfei Ji, ${ }^{3}$ Jian Sun, ${ }^{3}$ Jiabin Qian, ${ }^{1}$ and Xixiang Liu ${ }^{1}$ \\ ${ }^{1}$ School of Instrument Science and Engineering, Southeast University, Key Laboratory of Micro-Inertial Instrument \\ and Advanced Navigation Technology, Ministry of Education, Nanjing 210096, China \\ ${ }^{2}$ State Key Laboratory of Transient Optics and Technology, Xian Institute of Optics and Precision Mechanics, \\ Chinese Academy of Sciences, Xian 710119, China \\ ${ }^{3}$ Jiangsu Electrical Power Company Research Institute, Nanjing 211103, China
}

Correspondence should be addressed to Lihui Wang; wlhseu@163.com

Received 24 April 2014; Accepted 31 May 2014; Published 22 June 2014

Academic Editor: Ruqiang Yan

Copyright (C) 2014 Lihui Wang et al. This is an open access article distributed under the Creative Commons Attribution License, which permits unrestricted use, distribution, and reproduction in any medium, provided the original work is properly cited.

\begin{abstract}
To ensure low failure and high reliability of fiber optic current transducers (FOCTs), it is urgent to study methods of condition monitoring and fault diagnosis in FOCT. Faults in FOCT have statistical characteristics. With the analyzing of time domain and frequency domain features in fiber optic current transformers' measurement data, we establish correspondence between the physical characteristics of key components in transformer and data features and then build diagnostic analysis model based on Allan variance. According to the Allan variance calculation results, we can diagnose fiber optic current transformer's health state and realize faults location. Experiment results show that diagnostic methods based on Allan variance are accurate and effective to identify fault features.
\end{abstract}

\section{Introduction}

Fiber optic current transducers (FOCTs) are achieving increased acceptance and application in high voltage substations due to their superior accuracy, bandwidth, dynamic range, and inherent isolation. FOCTs are influenced by various factors such as electricity, heat, machinery, and environment in operation $[1,2]$, so their performance degrades gradually, which eventually leads to a fault. FOCTs are the basic components of the power system. Once they fail, this will cause local even wide area blackout, resulting in huge economic loss and social impact. At present, domestic and foreign maintenances on power equipment mostly in the regular offline maintenance state, which not only affects the normal work of the power grid but also causes its relatively low efficiency [3]. Thus, it is urgent to research on condition monitoring and fault diagnosis of FOCT.

According to the information about measured values by condition monitoring and their processing results, fault diagnosis of FOCT reasons, judges, finds out fault's types, location, and severity, and then puts forward proposals on equipment repair processing. Condition monitoring is the collection process of characteristic quantity [4]. It records, classifies, and evaluates the running state of the equipment. And it also provides decisions for equipment maintenance and repair. Fault diagnosis is the analysis and judgment process after characteristic quantity's collection, locating the occurred fault and judging the degree of fault development based on fault tag. Due to imperfect characteristics of the optical components in FOCT and environmental interference, there is a series of regular statistical noise in the output signal of FOCT. Random noises of FOCT come from light source coherent noises, light source intensity noises, photodetector shot noises, thermal noises in electronic devices, environmental noises, and time-varying noises because of device aging and other factors. Despite the large number of noise sources, output data of FOCT reflect that the characteristics mainly include angle random walk, bias instability, rate random walk, rate ramp, quantization noise, and sinusoidal noise.

As a complement to the frequency domain analysis, Allan variance is a time domain analysis technique originally 


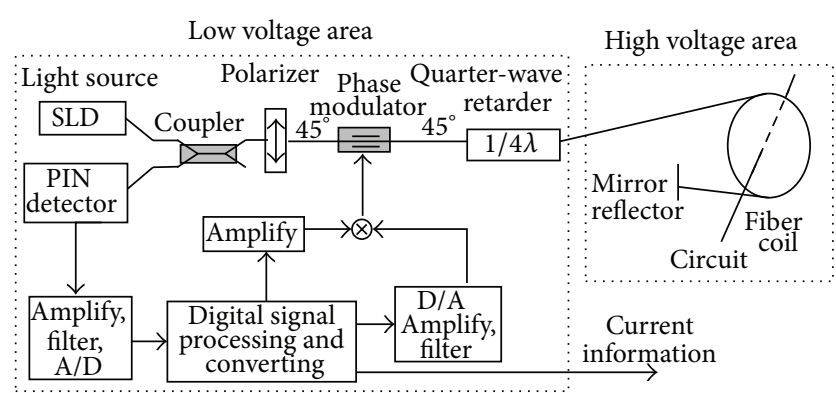

FIGURE 1: Structure of reflective FOCT with reflection mirror.

developed to study the frequency stability of oscillators [5-7]. It can be used to determine the character of the underlying random processes that give rise to the data noise. As such, it helps to identify the source of a given noise term present in the data, whether it is inherently in the instrument or in the absence of any plausible mechanism within the instrument, its origin should be sought in the test setup.

In this paper, we analyze the principle of Allan variance, evaluate types of random noise error, identify the statistical properties of various types of random errors, and locate noise error source. In the application of condition monitoring and fault diagnosis, we establish relational models between the noise characteristics and fault source with analysis of noise characteristics in time and frequency domain, respectively, according to the characteristics and trend of relevant parameters in FOCT. The model can monitor the health status of FOCT, judge fault position in fault state, provide the alarm when necessary, and finally provide the basis for further steps.

\section{Principle of Fault Diagnosis in FOCT}

2.1. Introduction of FOCT. FOCT is a kind of optical sensor based on Faraday magneto optical effects and optical interference theory $[8,9]$. It uses closed loop feedback system to measure the change of light intensity caused by optical nonreciprocal phase difference in real time, in order to acquire the information of measured current proportional to nonreciprocal phase. FOCT is composed of a wide spectrum light source, a polarization maintaining fiber, a polarizer, an electrooptical modulator, the signal processing unit, and so forth. There are multiple signal transmission and processing links, such as photoelectric conversion and electrooptical conversion. Output data of transducer have the random noise characteristics of FOCT.

According to the structure of fiber optic sensor head, FOCTs are divided into reflective FOCT and Sagnac FOCT $[2,9]$. The structure of FOCT with reflection mirror is shown in Figure 1; the structure of FOCT with fiber loop is shown in Figure 2.

Propagation process of light waves in reflective FOCT can be described as follows. The waves generated by the light source are polarized to linear polarized waves by polarizer after passing through the coupler. The linear polarized waves enter the polarization-maintaining fiber at $45^{\circ}$, transferring evenly into the polarization axis $X$ fiber (fast axis) and $Y$-axis

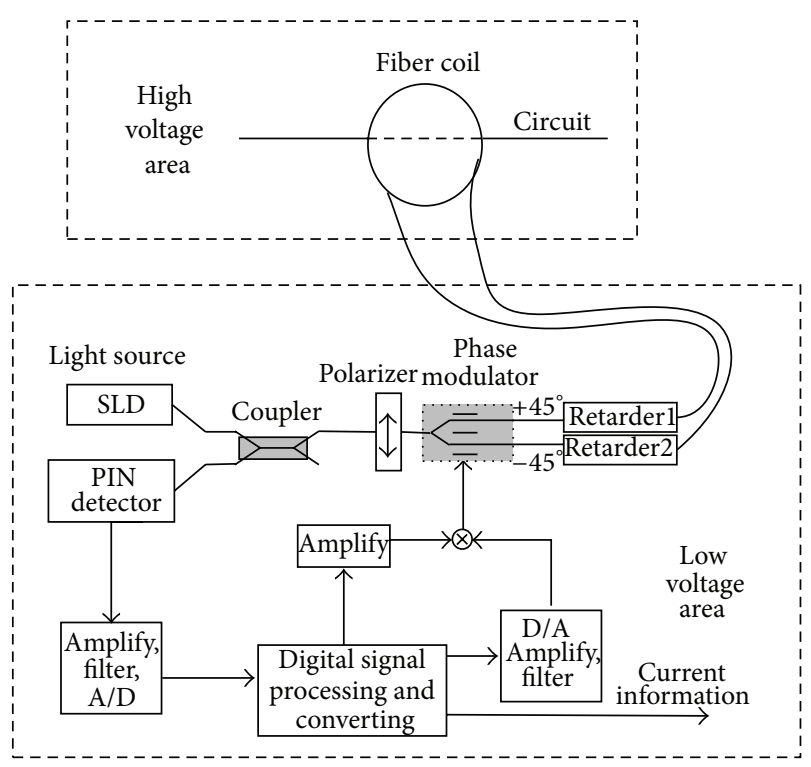

FIGURE 2: Structure of Sagnac FOCT with fiber loop.

(slow axis). Then, after passing through the $1 / 4 \lambda$ wave plate at $45^{\circ}$, the two beams of orthogonal mode waves are converted into left and right circular polarized waves, respectively, which enter the sensor fiber after that. In the sensor fiber, the two circular polarized waves transfer in different speeds due to Faraday field effect caused by current. After mirror reflected by sensor fiber's end face, they exchange their polarization modes (namely, the light of left-handed rotation changes into the light of right-handed rotation, and the light of right-handed rotation changes into the light of left-handed rotation.). They pass through the sensor fiber again and interact with the magnetic field generated by current again, doubling the phase. After passing through the $1 / 4 \lambda$ wave plate, the two beams of light revert to linear polarized waves. While they entered along the $X$-axis and $Y$-axis, they emit out wave plate along the $Y$-axis and $X$-axis of polarization-maintaining fiber, interfering in the polarizer. In the transmission process of the waves, the two beams of interfered waves pass through the $X$-axis and $Y$-axis of polarization-maintaining fiber as well as left-handed and right-handed rotation mode of sensor fiber, only different in time. Thus, the waves which return to detector only carry the nonreciprocal phase difference caused by Faraday effect.

Propagation process of light waves in Sagnac FOCT can be described as follows. The waves generated by the light source enter the integrated optical chip through the coupler and are polarized to linear polarized waves through the polarizer. The polarized waves are split into two parts at the beam splitter in the integrated optical chip, entering the wave plate, respectively, at plus or minus $45^{\circ}$ (clockwise light is $45^{\circ}$ and counterclockwise light is $45^{\circ}$ ). Prior to entering a low birefringence polarization-maintaining fiber optic sensor head in opposite directions, respectively, the polarized waves are converted into left and right circular polarized waves by $1 / 4 \lambda$ wave plate. Faraday field effect caused by current rotates the plane of polarization of two circular polarized waves. 
The waves are converted back into linear polarized waves when passing through another $1 / 4 \lambda$ wave plate and are brought back to interference. Rotation angles of polarization plane in two beams of interfered light are equal and opposite, then, interference phase in Sagnac FOCT is twice the Faraday phase shift. According to modulation technology of digital closed-loop signals, the information about the magnitude of current in measured power line can be acquired by detecting the phase difference of output lights.

2.2. Fault Features in FOCT. Although Sagnac FOCT and reflection FOCT are different in structure, they have the similar light waves sensing principle and signal processing steps. That means they all build digital closed-loop feedback system using high speed signal processing unit and electrooptic phase modulator in order to measure the information of nonreciprocal phase caused by Faraday magnetic field effect in real time and finally acquire the information of external current. Influenced by environmental factors and inherent factors, FOCTs reflect the same noise characteristics, which are mainly divided as the following categories $[5,10]$.

Bias instability (BI) noises reflect the bias low-frequency fluctuation of FOCT. BI noises originate from discharge assembly in FOCTs, plasma discharge, circuit noises, environmental noises, and many other components which can generate random flashing. It is useful to inhibit BI by reliability design of FOCTs and taking corresponding filtering method. Angle random walk (ARW) noises show the ultimate precision of FOCTs and are an important indicator to measure the IFOG noise level. Photon shot noises of photoelectric detector (PIN) in FOCT result in the uncertainty of Faraday phase shift measurement, which cause a limit of current measurement. Shot noises also cause current random fluctuation of current-voltage feedback impedance in PIN preamplifier, resulting in pseudo-Faraday phase shift. The phase shift influences IFOG minimum bias stability and decides FOCT's precision. ARW noises are the result of integrated broadband rate power spectral density, originating mainly from photodetector shot noises, amplifier noises, electronic device thermal noises, and some high frequency noises whose relevant time is shorter than sampling time. High frequency noises whose relevant time is shorter than sampling time can be eliminated. It is also efficient to inhibit ARW noises by using high-qualified light source and photoelectric detector and improving the stability of environmental temperature. Rate ramp (RR) is in essence a definite error, rather than a random error. The strength of light source in FOCT changes monotonously and very slowly and lasts for a long time, which causes RR noises. It is useful to reduce RR noises by ensuring the long-term stability of optoelectronic devices and working environment of FOCTs and determining the error compensation in the method of establishing the mathematical model. Rate random walk (RRW) noises reflect index correlated noises of long correlation time in limit condition. RRW noises are the integral result of phase value power spectral density, associated with long term effects of resonator. They are generated after white noises pass the integrator. RRW can be inhibited by reducing the aging effect of crystal oscillator. Sinusoidal noise (SN) is a kind of systematic error whose power spectral density is presented by several different frequencies. High frequency noises are generated by laser plasma oscillations in the discharge process; low frequency noises are caused by environmental periodic change. When the sinusoidal noises have sinusoidal waveform with multipeak, it is easier to show $\mathrm{SN}$ by the plot of power spectral density. Quantization noises (QN) reflect the minimum resolution of current's information of FOCTs. Sampling values of interference signals in FOCT are converted to digital quantities by $\mathrm{A} / \mathrm{D}$ and are sent into signal processor. In the measurement of time interval, measurement phase induced by current electromagnetic field is not integer times of quantified step size, while the amplitude of signals gets quantified over time, which causes quantization error. In the application environment with requirement of high sampling rate, large QN is caused, which can be reduced by improving the accuracy of acquisition system and shortening the initial sampling time.

2.3. Allan Variance Algorithm and Fault Diagnosis. Supposing that the current data with $\tau_{0}$ sampling period in FOCT is $N$-dimensional, we can obtain data collection $\left\{I(0), I\left(\tau_{0}\right), I\left(2_{\tau 0}\right), \ldots, I\left(N \cdot \tau_{0}\right)\right\}$. Allan variance is a time domain analysis technique based on function of time length. It can be used to calculate frequency data stability of FOCT in the time domain, analyze the random noise characteristic of FOCT, and isolate and identify the random error model and its parameter using the slope of the curve model in log-log plot $[5,6]$. The Allan variance is defined as follows:

$$
\sigma^{2}(\tau)=\frac{1}{2}\left\langle\left(\bar{I}_{k+m}(\tau)-\bar{I}_{k}(\tau)\right)^{2}\right\rangle
$$

where \langle\rangle is the ensemble average. $\bar{I}_{k+m}(\tau)=\left(w_{k+2 m}-\right.$ $\left.w_{k+m}\right) / \tau$ and $\bar{I}_{k}(\tau)=\left(w_{k+m}-w_{k}\right) / \tau$ are average current. $\tau=m \cdot \tau_{0}$ is correlation time. $\tau_{0}$ is the minimum sampling period. $w_{k}$ is the value of current for the moment of $k \tau_{0}$. Consider

$$
\begin{aligned}
\sigma^{2}(\tau) & =\frac{1}{2}\left\langle\left(\bar{I}_{k+m}(\tau)-\bar{I}_{k}(\tau)\right)^{2}\right\rangle \\
& =\frac{1}{2 \tau^{2}}\left\langle\left(w_{k+2 m}+w_{k}-2 w_{k+m}\right)^{2}\right\rangle .
\end{aligned}
$$

The Allan variance can be expressed as follows:

$$
\sigma^{2}(\tau)=\frac{1}{2 \tau^{2}(N-2 m)} \sum_{k=1}^{N-2 m}\left(w_{k+2 m}+w_{k}-2 w_{k+m}\right)^{2} .
$$

The Allan variance obtained by performing the prescribed operations is related to the PSD of the noise terms in the original data set. The relationship between Allan variance and the two-sided PSD $S_{E}(f)$ is given by the following equation:

$$
\sigma^{2}(\tau)=4 \int_{0}^{\infty} S_{E}(f) \cdot F(f) d f=4 \int_{0}^{\infty} S_{E}(f) \frac{\sin ^{4}(\pi f t)}{(\pi f t)^{2}} d f .
$$


Equation (4) indicates that Allan variance is proportional to the total energy of random noises when the random noises pass through the filter of function $F(f)$. Filter of band pass depends on the correlation time $\tau$. By adjusting it (namely, adjusting band filter), different types of stochastic processes can be detected. The credibility of Allan variance estimation improves with the increasing numbers of independent sets.

Equation (4) is the key result that will be used throughout to characterize the rate noise PSD from the Allan variance calculations. Its physical interpretation is that the Allan variance is proportional to the total noise power of the FOCT current output when passed through a filter with the transfer function of $F(f)$. This particular transfer function is the result of the method used to create and operate on the clusters. It is seen from (4) that the filter of band pass depends on $\tau$. This suggests that different types of random processes can be examined by adjusting the filter of band pass, namely, by varying $\tau$. Thus the Allan variance provides a means of identifying and quantifying various noise terms that exist in the data. It is normally plotted as the square root of the Allan variance versus $\tau[\sigma(\tau)]$, on a log-log plot. The following paragraphs show the application of (4) to a number of noise terms that are either known to exist in the FOCT or otherwise influence its data. Power spectral density of random noise in FOCT has the following features.

For angle random walk, the associated rate PSD is represented by the following equation:

$$
S_{E}(f)=N^{2}
$$

where $N$ is the angle random walk coefficient.

The following equation is obtained by performing integration:

$$
\sigma^{2}(\tau)=\frac{N^{2}}{\tau}
$$

For bias instability, the associated rate PSD is represented by the following equation:

$$
\begin{aligned}
& S_{E}(f)=\left(\frac{B^{2}}{2 \pi}\right) \frac{1}{f}, \quad f \leq f_{0}, \\
& S_{E}(f)=0, \quad f>f_{0},
\end{aligned}
$$

where $B$ is the bias instability coefficient and $f_{0}$ is the cut-off frequency.

For rate random walk, the associated rate PSD is represented by the following equation:

$$
S_{E}(f)=\left(\frac{K}{2 \pi}\right)^{2} \frac{1}{f^{2}},
$$

where $K$ is the rate random walk coefficient.

For quantization noise, the associated rate PSD is represented by the following equation:

$$
S_{E}(f)=\tau Q^{2}
$$

where $Q$ is the quantization noise coefficient.

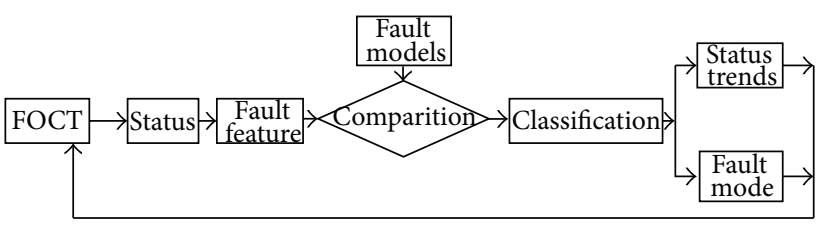

FIGURE 3: Structure of fault diagnosis system in FOCT.

Different error terms of random noises in FOCT appear in different correlation time domain. It is different in the power spectral density and the function relationship with correlation time $\tau$ of different noises. Assuming that the noise error term is independent in the statistical sense, integrated Allan variance $\sigma_{\text {total }}^{2}(\tau)$ is given by the following equation:

$$
\sigma_{\text {total }}^{2}(\tau)=\sum_{n=-2}^{2} A_{n} \tau^{n},
$$

where $A_{n}(n=-2,-1,0,1,2)$ correspond to quantization noise, angle random walk, bias stability, rate random walk and rate ramp, and many other coefficients of fitted polynomial related to noise, respectively. Consider

$$
\begin{aligned}
\sigma_{\text {total }}^{2}(\tau)= & \sum_{n=-2}^{2} A_{n} \tau^{n}=\frac{3 Q^{2}}{\tau^{2}}+\frac{N^{2}}{\tau} \\
& +\frac{2 \ln 2}{\pi} B^{2}+\frac{K^{2}}{3} \tau+\frac{R^{2}}{2} \tau^{2} .
\end{aligned}
$$

In (11), $Q$ is quantization noise coefficient; $N$ is angle random walk coefficient, $B$ is bias instability coefficient; $K$ is rate random walk coefficient; $R$ is rate ramp coefficient. Each type of noise errors corresponds to different slope in the Allan variance correlation time log-log plot. The slope of quantization noise, angle random walk, bias stability, rate random walk, and rate ramp is $-1,-0.5,0,0.5$ and 1 , respectively $[5,6]$.

Allan variance analysis method and the modeling technology can effectively separate and identify several main types of random noises which influence FOCT's precision. By analyzing the curve, the corresponding noise error values can be estimated, making a comprehensive evaluation of overall performance of FOCT. In addition, error source of FOCT's noise error can be located by analyzing different types of noise values. It is not only effective to improve the performance of FOCT, but also convenient to identify and locate fault in FOCT.

Combining FOCT state parameters and available information, we can build an intelligent diagnosis system based on some algorithms, to determine the status of the device with theoretical derivation and realize fault detection and diagnosis [11-13]. The structure of intelligent diagnosis system is shown in Figure 3. Figure 4 shows fault diagnosis process in FOCT. We can obtain feature vectors in time domain and frequency domain with signal processing model and then detect the failure source by using the mapping relationship between feature vectors and failure source. 


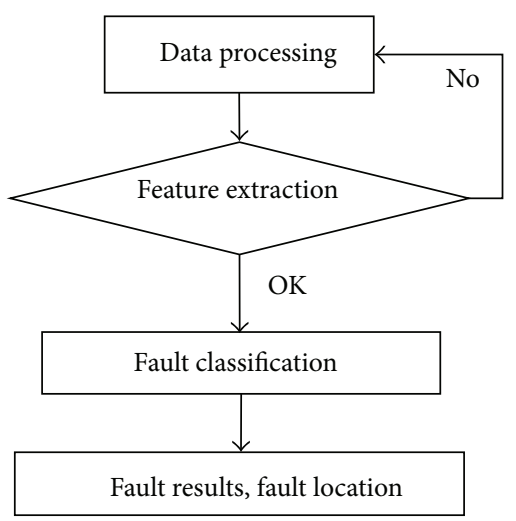

FIGURE 4: Flow chart of fault diagnosis in FOCT.

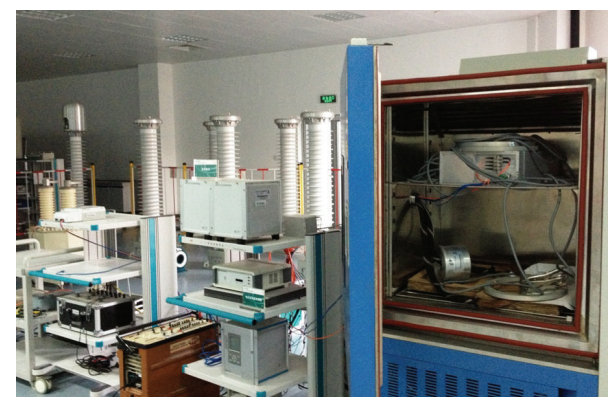

FIGURE 5: Experimental environment of current transducer test system.

\section{Experiments}

To verify the performance and fault diagnosis methods of fiber optic current transducer, we built a current transformer test system, as shown in Figure 5. Figure 6 shows the principle of current transformer test system. FOCT, standard current transducer $(\mathrm{CT})$, and current generator are stringed in the same current loop; the current generator applies primary current to the two sets of CT, while different error factors are added to FOCT. The function of transducer calibration part is to process data in FOCT and reference standard CT and to calculate the measurement deviation. The function of fault diagnosis part is to monitor FOCT's operation status and to diagnose FOCT's fault by using Allan variance methods.

We test fault characteristics of several fiber optic current transformers, respectively. Figure 7 shows sample of current measurements data in one FOCT. Allan variance log-log curve expresses the random error in fiber current transformer accurately, including angle random walk, bias instability, rate random walk, rate ramp, quantization noise, and sinusoidal noise. Theoretically, the slope of quantization noise, angle random walk, bias stability, rate random walk, and rate ramp is $-1,-0.5,0,0.5$, and 1 , respectively. Based on characteristics of random error, we can evaluate health state of FOCT; moreover, error source in FOCT is located. Figure 8 shows Allan variance analysis curve of the first FOCT. We can find that quantization noise with rate ramp of -1 , angle random walk with rate ramp of $-1 / 2$, and bias instability noise with

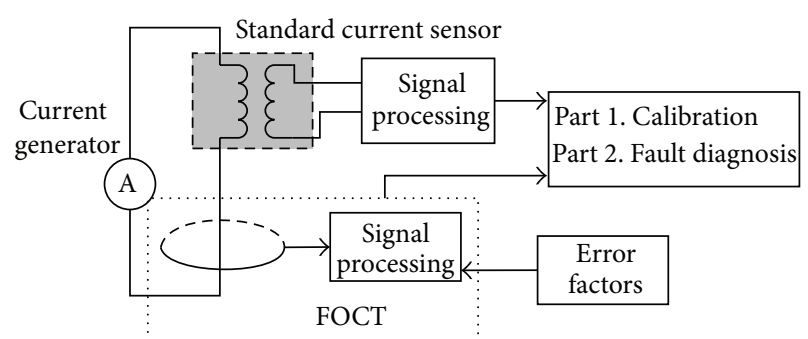

FIGURE 6: Principle block of current transducer test system.

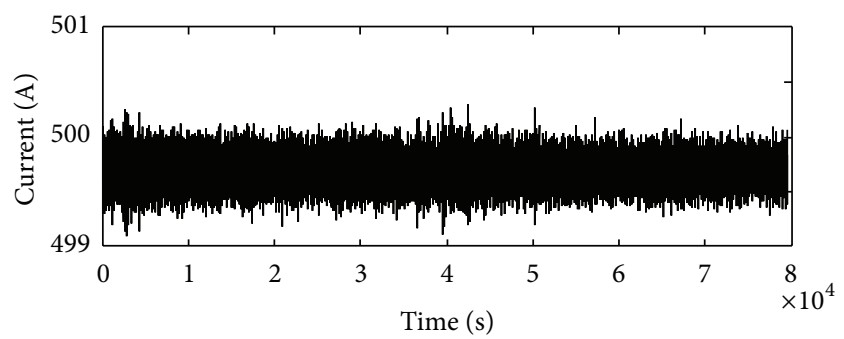

Figure 7: Current measurements data in FOCT.

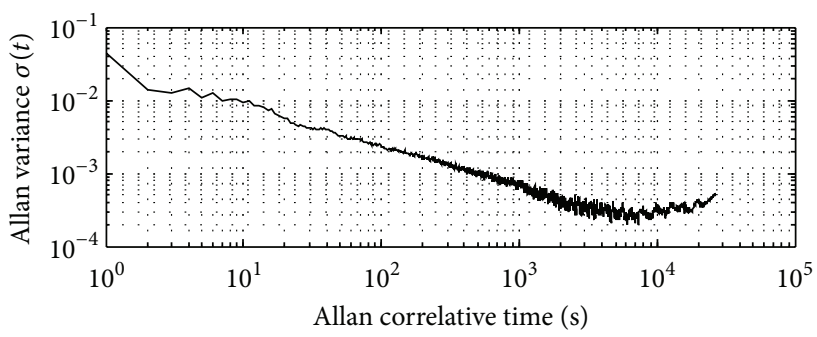

Figure 8: Allan variance curve of first FOCT.

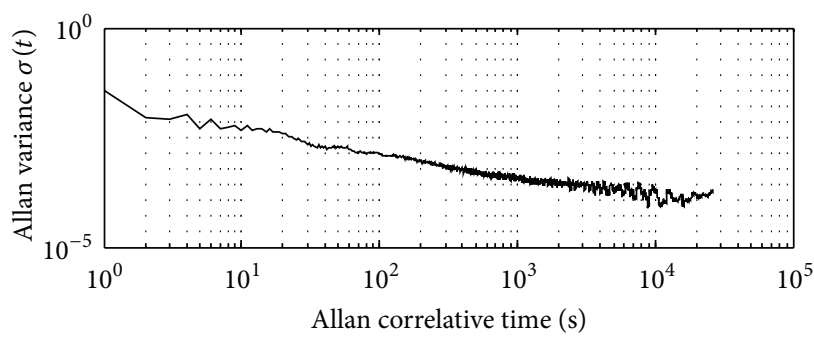

FIGURE 9: Allan variance curve of second FOCT.

rate ramp of 0 are the main errors in FOCT; moreover, we can locate noise source, mainly from photon shot noise in detector, amplifier noise, thermal noise electronics, and high frequency noise. Minimum resolution performance of this FOCT is affected. Figure 9 shows Allan variance analysis curve of the second FOCT. We can find that angle random walk with rate ramp of $-1 / 2$ and sinusoidal noise are the main errors in FOCT, and high-frequency noise dominated system noise is the main noise source. 


\section{Conclusions}

Allan variance can be used to analyze the time domain characteristics of the underlying random processes in FOCT. It helps to identify the source of a given noise term present in the data, whether it is inherently in FOCT or in the absence of any plausible mechanism within FOCT. In this paper, we verified the validity of the Allan variance methods in soft fault diagnosis through theoretical analysis and fault simulation experiments. Allan variance is suitable for evaluation and diagnosis of soft fault in FOCT, which can avoid potential failures, such as performance fault induced by temperature, light source, and other factors. For abrupt-changing fault, we can compare and judge the fault characteristics with feature model by using wavelet transform methods and then decide whether the fault is from FOCT or grid failure.

\section{Conflict of Interests}

The authors declare that there is no conflict of interests regarding the publication of this paper.

\section{Acknowledgments}

The project is supported by the following funds. Fundamental Research Funds for the Central Universities (2242013R30016), Natural Science Foundation of Jiangsu Province (BK2012326, BK20130099), National Natural Science Foundation of China (61203192), Research Fund of China Ship 8 Industry (13J3.8.4), and Foundation of Key Laboratory of Micro-Inertial Instrument and Advanced Navigation Technology, Ministry of Education (201103).

\section{References}

[1] K. Bohnert, P. Gabus, J. Kostovic, and H. Brändle, "Optical fiber sensors for the electric power industry," Optics and Lasers in Engineering, vol. 43, no. 3-5, pp. 511-526, 2005.

[2] J. Blake, P. Tantaswadi, and R. T. de Carvalho, "In-line sagnac interferometer current sensor," IEEE Transactions on Power Delivery, vol. 11, no. 1, pp. 116-121, 1996.

[3] H.-B. Wang, K.-M. Tang, R.-L. Xu, X.-J. Zhu, and X.-J. Li, "Diagnosis of soft fault of electronic transformer in digital substation," Power System Protection and Control, vol. 40, no. 24, pp. 53-58, 2012.

[4] S. N. Huang and K. K. Tan, "Fault detection, isolation, and accommodation control in robotic systems," IEEE Transactions on Automation Science and Engineering, vol. 5, no. 3, pp. 480489, 2008.

[5] "IEEE standard specification format guide and test procedure for single-axis laser gyros," Tech. Rep. IEEE Std 647-2006, IEEE Aerospace and Electronic Systems Society.

[6] "IEEE standard specification format guide and test procedure for single-axis interferometric fiber optic gyross," Tech. Rep. IEEE Std 952-1997, Gyro and Accelerometer Panel of the IEEE Aerospace and Electronic Systems Society.

[7] D. W. Allan and J. A. Barnes, "A modified Allan variance with increased oscillator characterization ability," in Proceedings of the 35th Annual Frequency Control Symposium, vol. 5, pp. 470475, 1981.
[8] K. Bohnert, P. Gabus, J. Nehring, and H. Brändle, “Temperature and vibration insensitive fiber-optic current sensor," Journal of Lightwave Technology, vol. 20, no. 2, pp. 267-276, 2002.

[9] K. Bohnert, P. Gabus, J. Nehring, H. Brändle, and M. G. Brunzel, "Fiber-optic current sensor for electrowinning of metals," Journal of Lightwave Technology, vol. 25, no. 11, pp. 3602-3609, 2007.

[10] N. Zhang and X. Li, "Research on theoretical improvement of dynamic Allan variance and its application," Acta Optica Sinica, vol. 31, no. 11, Article ID 1106003, 2011.

[11] K. Bouibed, A. Aitouche, and M. Bayart, "Sensor and actuator fault detection and isolation using two model based approaches: application to an autonomous electric vehicle," in Proceedings of the 18th Mediterranean Conference on Control \& Automation (MED '10), pp. 1290-1295, Marrakech, Morocco, June 2010.

[12] K. Bouibed, A. Aitouche, and M. Bayart, "Nonlinear parity space applied to an autonomous vehicle," Journal of Energy and Power Engineering, vol. 3, no. 12, pp. 10-18, 2009.

[13] A.-J. Khalid, J. Wang, and M. Nurudeen, "A new fault classification model for prognosis and diagnosis in CNC machine," in Proceedings of the 25th Chinese Control and Decision Conference (CCDC '13), pp. 3538-3543, May 2013. 


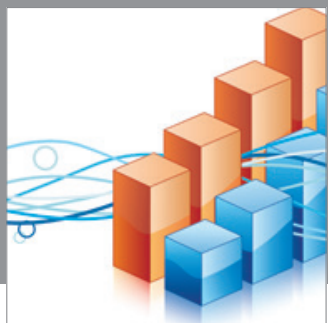

Advances in

Operations Research

mansans

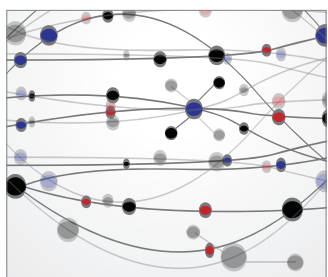

The Scientific World Journal
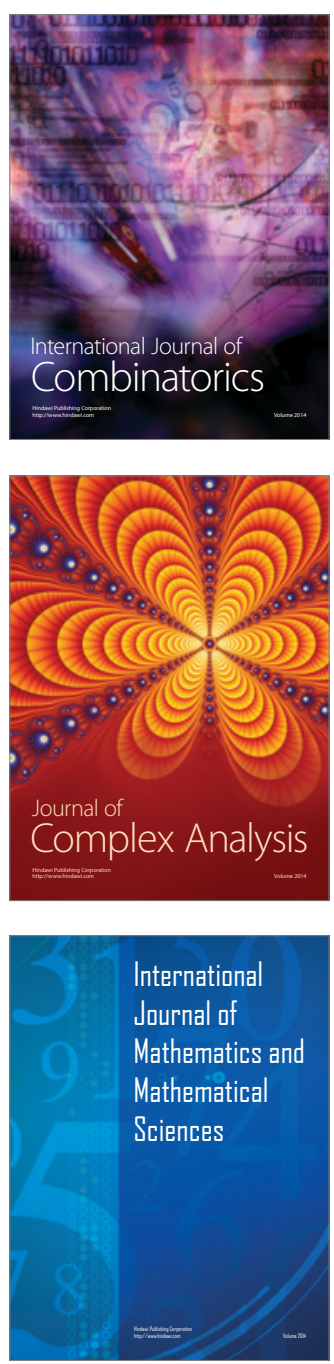
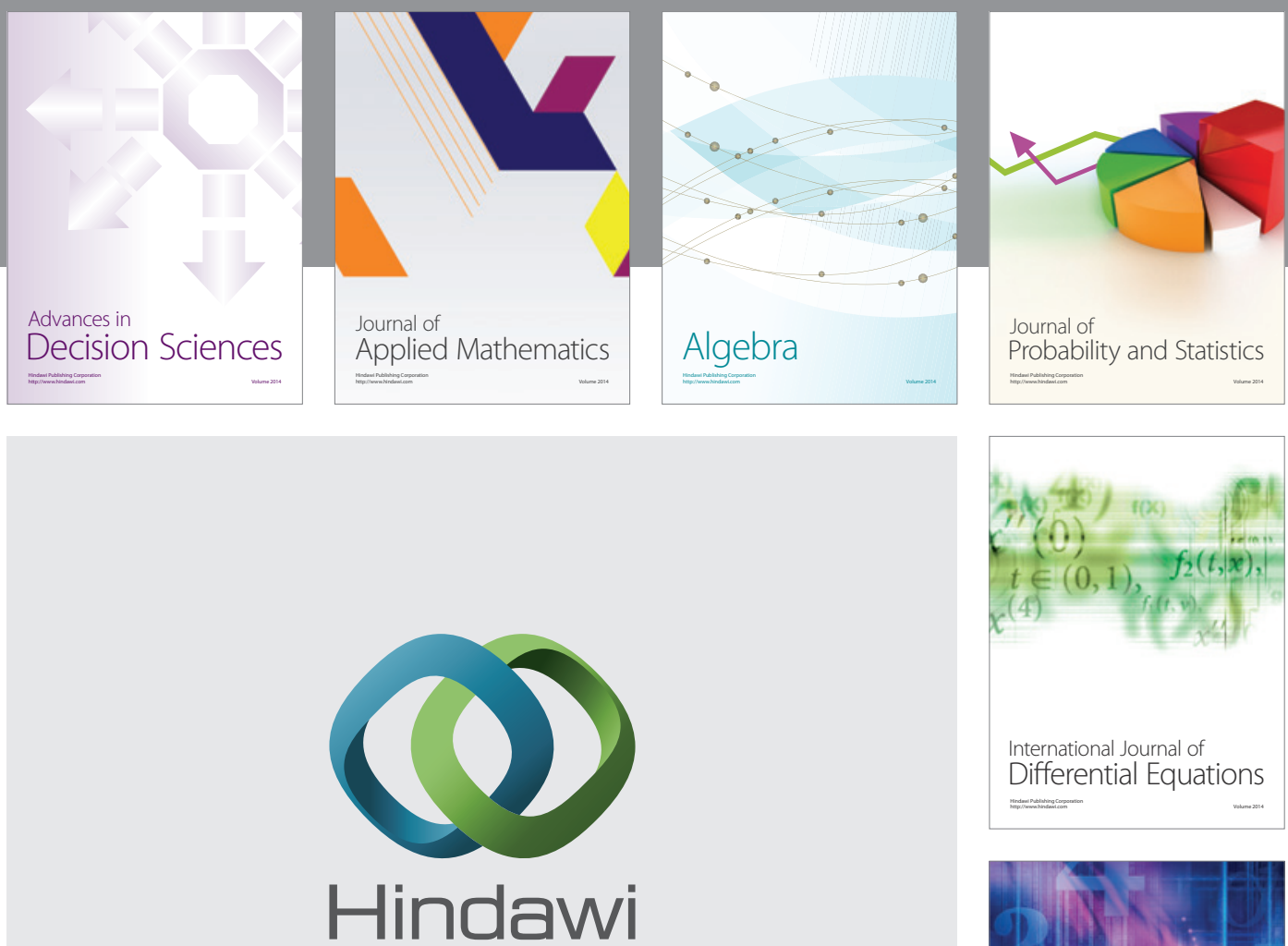

Submit your manuscripts at http://www.hindawi.com
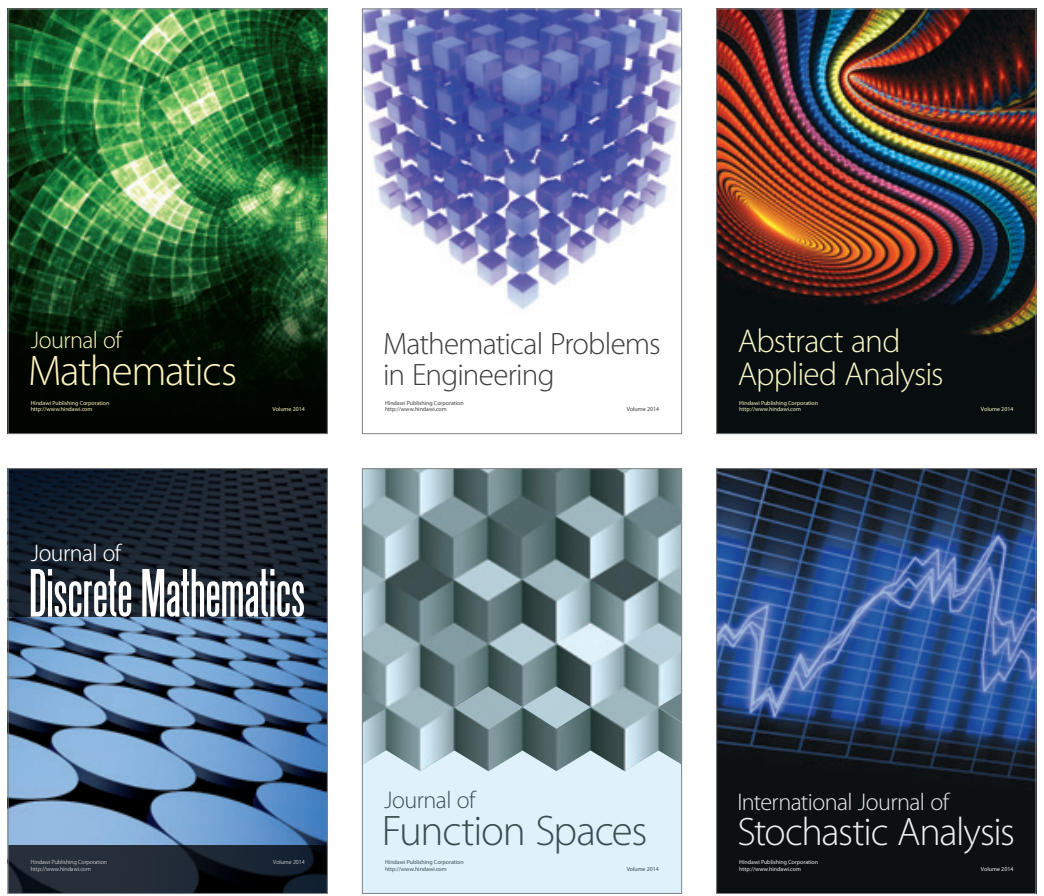

Journal of

Function Spaces

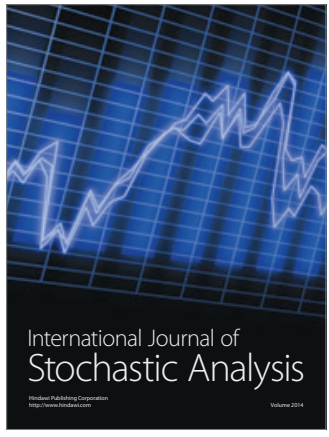

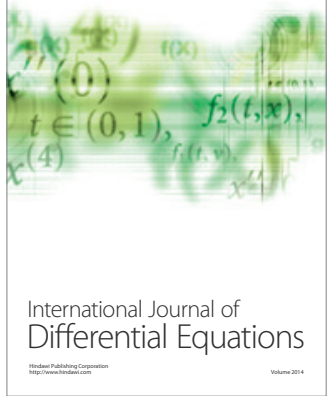
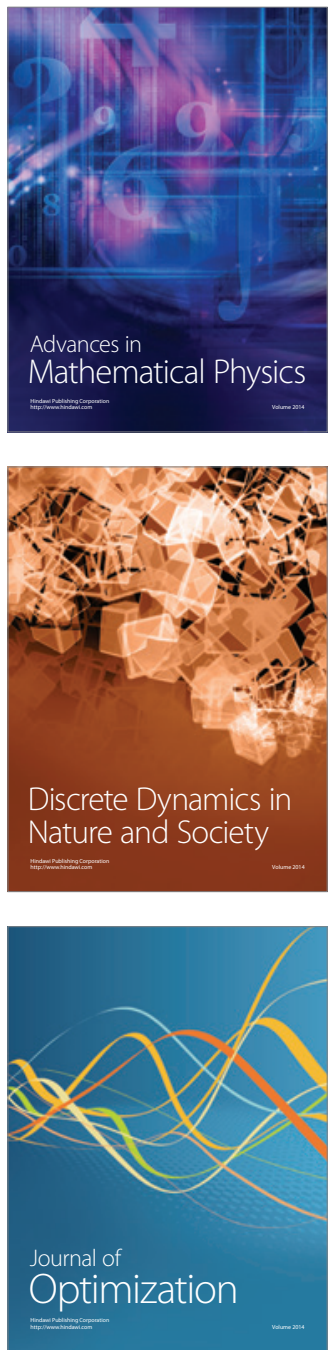